\title{
WAPD-T-Z Z1/9
}

BRIEF COMMUNICATION

MEASUREMENTS OF INTERFACIAL AREA CONCENTRATION IN TWO-PHASE FLOW WITH TWO-POINT CONDUCTIVITY PROBE

\author{
Q. WU ${ }^{1}$, D. ZHENG ${ }^{1}$, M. ISHI ${ }^{1}$ and S.G. BEUS ${ }^{2}$ \\ Thermal Hydraulics and Reactor Safety Laboratory \\ ${ }^{1}$ School of Nuclear Engineering, Purdue University, West Lafayette, IN 47907, U.S.A. \\ ${ }^{2}$ Bettis Atomic Power Laboratory, Westinghouse Electric Corporation, \\ Box 79, West Mufflin, PA 15122, U.S.A.
}

Kataoka, Ishii and Serizawa (1986 [1]) analyzed the measurements of the local time-averaged interfacial area concentration in two-phase flow with a two-point conductivity probe. They considered the influence of the bubble velocity fluctuation on the measurement and directly transferred the mathematics concept of the local time-averaged interfacial area concentration into the measurable parameters. In the end of the derivation, however, the expression of the interfacial area concentration was inappropriate due to the over-simplification to the integration limits of the probability distributions. Consequently, the resultant interfacial area concentration may be significantly lower than the actual value. Since the formula is very important for the interpretation of experimental data, we feel it is necessary to provide a correction to the original work.

According to Ishii (1975 [2]), the definition of the time-averaged interfacial area concentration is given by

$$
\bar{a}^{t}=\frac{1}{\Delta t} \sum_{j} \frac{1}{\mathbf{V}_{i j} \cdot \mathbf{n}_{j}}
$$

where $\mathbf{j}$ denotes the $\mathbf{j}$-th interface, $\mathbf{V}_{\mathbf{i}}$ and $\mathbf{n}$ are the velocity and normal vector of the bubble interface as shown in Fig. 1, and $\Delta t$ is the time interval for time averaging. If there is no

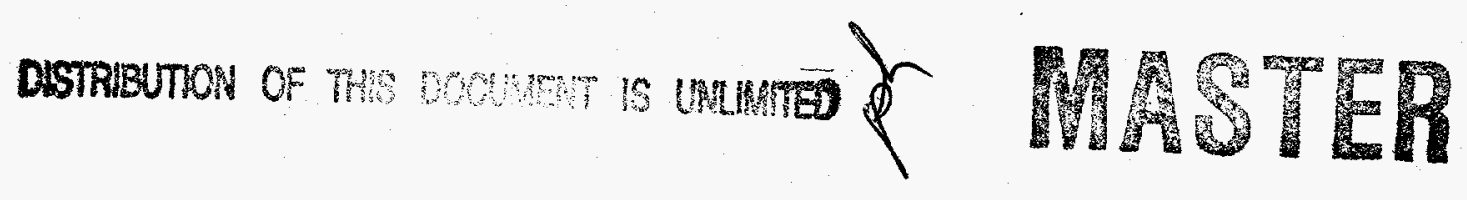




\section{DISCLAIMER}

Portions of this document may be illegible in electronic image products. Images are produced from the best available original document. 


\section{DISCLAIMER}

This report was prepared as an account of work sponsored by an agency of the United States Government. Neither the United States Government nor any agency thereof, nor any of their employees, make any warranty, express or implied, or assumes any legal liability or responsibility for the accuracy, completeness, or usefulness of any information, apparatus, product, or process disclosed, or represents that its use would not infringe privately owned rights. Reference herein to any specific commercial product, process, or service by trade name, trademark, manufacturer, or otherwise does not necessarily constitute or imply its endorsement, recommendation, or favoring by the United States Government or any agency thereof. The views and opinions of authors expressed herein do not necessarily state or reflect those of the United States Government or any agency thereof. 
correlation between $V_{i}$ and $\phi$, the angle between the velocity and the normal vector, Eq. (1) can be modified in the following form,

$$
\bar{a}^{t}=2 N_{i} \overline{\frac{1}{V_{i} \cos \phi}}=2 N_{t} \frac{\overline{1}}{V_{i}} \cdot \frac{\overline{1}}{\cos \phi},
$$

where $\mathrm{N}_{t}$ is the total number of bubbles passing through the measuring point in a time interval of $\Delta \mathrm{t}$. The factor 2 implies that a bubble has two interfaces (upper and lower) in the probe direction. With a two-point conductivity probe [1] to measure the interface velocity in the $\mathrm{z}$ direction, the normal vector, $\mathbf{n}$, and the interface velocity, $\mathbf{V}_{\mathrm{i}}$, at the point where the probe hits the bubble are defined as

$$
\begin{aligned}
& \mathbf{n}=\sin \mu \sin v \hat{i}+\sin \mu \cos v \hat{j}+\cos \mu \hat{k} \\
& \mathbf{V}_{i}=V_{i}(\sin \alpha \sin \beta \hat{i}+\sin \alpha \cos \beta \hat{j}+\cos \alpha \hat{k})
\end{aligned}
$$

The angles in the above equations are defined in Fig. 2 (a) and (b). Thereby, the velocity measured with the two-point probe satisfies

$$
V_{z}=\frac{\Delta S}{\Delta t}=V_{i} \frac{\cos \phi}{\cos \mu}
$$

with $\Delta S$ as the distance between the two probe tips along the $\mathrm{z}$ direction. Since the amplitude of the bubble velocity is mainly determined by the local flow structure independent of $\phi$ and $\mu$, the following average can be obtained.

$$
\overline{\frac{1}{V_{z}}}=\overline{\frac{\cos \mu}{V_{i} \cos \phi}}=\overline{\frac{1}{V_{i}}} \cdot \frac{\overline{\cos \mu}}{\cos \phi} \text {. }
$$

Consequently, the time-averaged interfacial area concentration is given by 


$$
\bar{a}^{t}=2 N_{t} \frac{\overline{1}}{V_{z}} \cdot \overline{\frac{1}{\cos \phi}} \cdot\left(\overline{\frac{\cos \mu}{\cos \phi}}\right)^{-1},
$$

where $\cos \phi$ needs to be replaced in the form of

$$
\cos \phi=\mathbf{n}_{\mathbf{v}_{i}} \cdot \mathbf{n}=\cos \mu \cos \alpha+\sin \mu \sin \alpha \cos (v-\beta) .
$$

If the sample size is sufficiently large within a reasonably long time interval, Eq. (7) can be presented in terms of the measured velocities of interfaces and the probability density functions,

$$
\bar{a}^{t}=2 N_{t}\left\{\frac{\sum_{j} \frac{1}{V_{z j}}}{\sum_{j}}\right\} \frac{\iiint \frac{P(\alpha, \mu,(v-\beta)) d \alpha d \mu d(v-\beta)}{(\cos \alpha \cos \mu+\sin \alpha \sin \mu \cos (v-\beta))}}{\iiint \frac{P(\alpha, \mu,(v-\beta)) \cos \mu d \alpha d \mu d(v-\beta)}{(\cos \alpha \cos \mu+\sin \alpha \sin \mu \cos (v-\beta))}},
$$

where, $P(\alpha, \mu,(v-\beta))$ is the probability density function, and the summation only counts the front velocities of the bubbles. In the article of Kataoka et al., by assuming spherical interfaces and an equal probability for a probe to pass every part of the interface, the probability function was thus given by

$$
P(\alpha, \mu,(v-\beta)) d \alpha d \mu d(v-\beta)=g(\alpha) \frac{1}{\pi} \cos \mu \sin \mu d \mu d(v-\beta) d \alpha .
$$

For the probability density function of the angle $\alpha, g(\alpha)$, a simple linear distribution was proposed as $g(\alpha)=1 / \alpha_{0}$. With the integration intervals of $0 \leq \alpha \leq \alpha_{0}, 0 \leq \mu \leq \pi / 2$, and $0 \leq(\nu-\beta) \leq 2 \pi$, the integrations were carried out and Eq. (9) was simplified as

$$
\bar{a}^{t}=2 N_{t}\left\{\frac{\sum_{j} \frac{1}{V_{z j}}}{\sum_{j}}\right\} \frac{2}{\int_{0}^{\alpha_{0}}\left(1 / \alpha_{0}\right) \sin ^{2} \alpha \ln ((1+\cos \alpha) / \sin \alpha) d \alpha} \frac{\sin \alpha_{0}\left(1 / \alpha_{0}\right)}{0} .
$$


The rest of the integration in the above equation is difficult to be carried out analytically. A numerical approach seems necessary. In the original paper of Kataoka et al., an exponent 2 over $\sin \alpha$ was missed, and therefore an analytical solution for the integration was carried out.

However, for a bubble with velocity in an arbitrary $\alpha$ direction, the probe tip can only hit the upper hemisphere divided by the shaded area as shown in Fig. 3. With a fixed angle ( $v-\beta)$, the integration region of $\mu$ should be $0 \leq \mu \leq \mu_{\max }(\alpha,(\nu-\beta))$ rather than $0 \leq \mu \leq \pi / 2$. Here, $\mu_{\max }$ is a function of $\alpha$ and $(\nu-\beta)$, which is obtained by simple geometric analysis.

$$
\mu_{\max }=\frac{\pi}{2}+2 \arcsin \left[\cos (v-\beta) \sin \left(\frac{\alpha}{2}\right)\right] .
$$

The corresponding probability density function should be

$$
P(\alpha, \mu,(v-\beta)) d \alpha d \mu d(\nu-\beta)=g(\alpha) \cdot \frac{1}{\pi} \cos \left(\mu_{\max }-\mu\right) \sin \left(\mu_{\max }-\mu\right) d \mu d(\nu-\beta) d \alpha,
$$

which assumes that the interfaces are composed of spherical bubbles and the probe passes every part of the shaded surface in Fig. 3 with equal probability. By substituting Eq. (13) into Eq. (9), the time-averaged interfacial area concentration is obtained as

$$
\begin{aligned}
& \bar{a}^{t}=2 N_{t}\left\{\frac{\sum_{j} \frac{1}{V_{z j}}}{\sum_{j}}\right\} I\left(\alpha_{0}\right), \\
& I\left(\alpha_{0}\right)=\frac{\int_{0}^{\alpha}\left\{\int_{0}^{2 \pi}\left[\int_{0}^{\mu_{\max }} \frac{g(\alpha) \sin \left(\mu_{\max }-\mu\right) \cos \left(\mu_{\max }-\mu\right)}{\pi(\cos \alpha \cos \mu+\sin \alpha \sin \mu \cos (v-\beta))} d \mu\right] d(v-\beta)\right\} d \alpha}{\int_{0}^{\alpha_{0}}\left\{\int_{0}^{2 \pi}\left[\int_{0}^{\mu_{\max }} \frac{g(\alpha) \cos \mu \sin \left(\mu_{\max }-\mu\right) \cos \left(\mu_{\max }-\mu\right)}{\pi(\cos \alpha \cos \mu+\sin \alpha \sin \mu \cos (v-\beta))} d \mu\right] d(v-\beta)\right\} d \alpha} .
\end{aligned}
$$


The analytical solution of the integration, $I\left(\alpha_{0}\right)$, is likely not possible. By proposing a simple flat distribution of $g(\alpha)=1 / \alpha_{0}$, the numerical solutions of $I\left(\alpha_{0}\right)$ for different values of $\alpha_{0}$ are presented in Fig. 4. Compared with the original solutions (Eq. (11)) of Kataoka et al, large discrepancies are observed in Fig. 4. The important difference is that the present integrations yield a $I\left(\alpha_{0}\right)$ slightly larger than 2 , whereas the original result was less than 2 . Notice that $I\left(\alpha_{0}=\right.$ 0 ) is supposed to be 2 if the bubbles move vertically in the $\mathrm{z}$ direction without lateral velocity components.

To avoid the complicated and inconvenient integrations, we introduce a new coordinate system as shown in Fig. 5, where the $\mathrm{z}$ direction is chosen in the velocity direction. The fluctuation of the angle between the probe direction and the bubble velocity is treated as if the probe randomly poked the bubbles with an angle $\alpha$. In such a case, the probe direction vector is given by (Fig. 6(a))

$$
\mathbf{n}_{\mathrm{P}}=\sin \alpha \sin \theta \hat{i}+\sin \alpha \cos \theta \hat{j}+\cos \alpha \hat{k}
$$

and the normal vector of the interface is defined as (Fig. 6(b))

$$
\mathbf{n}=\sin \phi \sin \psi \hat{i}+\sin \phi \cos \psi \hat{j}+\cos \phi \hat{k}
$$

The angle between the surface normal and the probe direction is $\mu$, and

$$
\cos \mu=\mathbf{n}_{\mathrm{P}} \cdot \mathbf{n}=\cos \phi \cos \alpha+\sin \phi \sin \alpha \cos (\psi-\theta)
$$

Consequently, according to Eq. (7), the time-averaged interfacial area concentration should be

$$
\bar{a}^{t}=2 N_{t}\left\{\frac{\sum_{j} \frac{1}{V_{z j}}}{\sum_{j}}\right\} \frac{\iiint \frac{P(\alpha, \phi,(\psi-\theta)) d \alpha d \phi d(\psi-\theta)}{\cos \phi}}{\iiint \frac{(\cos \alpha \cos \phi+\sin \alpha \sin \phi \cos (\psi-\theta)) P(\alpha, \phi,(\psi-\theta)) d \alpha d \phi d(\psi-\theta)}{\cos \phi}}
$$


The integration intervals are $0 \leq \alpha \leq \alpha_{0}, 0 \leq \phi \leq \pi / 2$, and $0 \leq(\psi-\theta) \leq 2 \pi$. Again, by assuming that the interfaces are composed of spherical bubbles and the probe passes every part of the upper hemisphere divided by the shaded area in Fig. 6 with equal probability, the probability density function is obtained as

$$
P(\alpha, \phi,(\psi-\theta)) d \alpha d \phi d(\psi-\theta)=g(\alpha) \frac{1}{\pi} \cos \phi \sin \phi d \phi d(\psi-\theta) d \alpha,
$$

Accordingly, the time-averaged interfacial concentration is given by

$$
\begin{aligned}
& \bar{a}^{t}=2 N_{t}\left\{\frac{\sum_{j} \frac{1}{V_{z j}}}{\sum_{j}}\right\} I\left(\alpha_{0}\right), \\
& I\left(\alpha_{0}\right)=\frac{\int_{0}^{\alpha_{0}}\left\{\int_{0}^{\pi / 2}\left[\int_{0}^{2 \pi} g(\alpha) \frac{\sin \phi}{\pi} d(\psi-\theta)\right] d \phi\right\} d \alpha}{\int_{0}^{\alpha_{0}}\left\{\int_{0}^{\pi / 2}\left[\int_{0}^{2 \pi} g(\alpha) \frac{\sin \phi(\cos \alpha \cos \phi+\sin \alpha \sin \phi \cos (\psi-\theta))}{\pi} d(\psi-\theta)\right] d \phi\right\} d \alpha} .
\end{aligned}
$$

If the probability density function $\mathrm{g}(\alpha)$ is assumed to be $1 / \alpha_{0}$, the integration, $\mathrm{I}\left(\alpha_{0}\right)$, can be readily carried out as

$$
I\left(\alpha_{0}\right)=2 \frac{\alpha_{0}}{\sin \alpha_{0}} .
$$

The numerical comparison of Eq. (23) and (15) is shown in Fig. 7. Fairly good agreement is obtained.

As a conclusion, the time-averaged interfacial area concentration measured by a two-point conductivity probe should be 


$$
\bar{a}^{t}=4 N_{t} \frac{\alpha_{0}}{\sin \alpha_{0}}\left\{\frac{\sum_{j} \frac{1}{V_{z j}}}{\sum_{j}}\right\},
$$

where the maximum angle between the bubble velocity and the probe direction is mainly determined by the turbulent fluctuation as pointed out in the original paper. Since $\alpha_{0}$ is usually small, less than 0.39 rad according to the data of Serizawa et al. [3], the effects of the angle are negligibly small, which may add a maximum of $3 \%$ to the value obtained without considering the lateral velocity of the bubbles. This is quite different from Kataoka's conclusion, which yields a modification factor much less than one for the case without lateral velocity components. 


\section{REFERENCES}

1. KaTAOKA, I., IsHII, M., \& SeRIzAWA, A. 1986 Local formulation and measurements of interfacial area concentration in two-phase flow. Int. J. Multiphase Flow Vol. 12 No. 4, pp. $505-529$.

2. ISHII, M. 1975 Thermal-fluid Dynamic Theory of Two-phase Flow. Eyrolles, Paris/Scientific and Medical Publications of France, New York.

3. SERIZAWA, A., KATAOKA, I., \& MICHIYOSHI, I. 1975 Turbulent structure of air-water bubbly flow II. Local properties. Int. J. Multiphase Flow 2, pp. 235-246. 


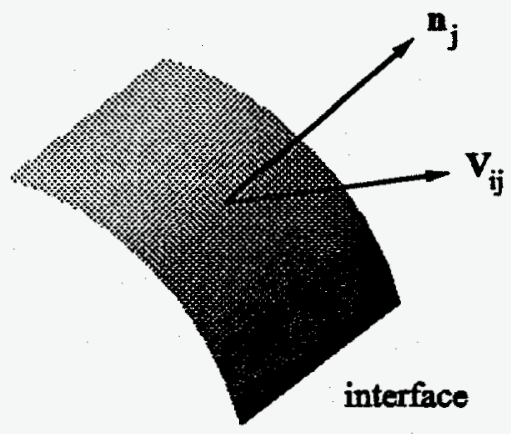

Fig. 1 Surface normal vector and interface velocity vector 


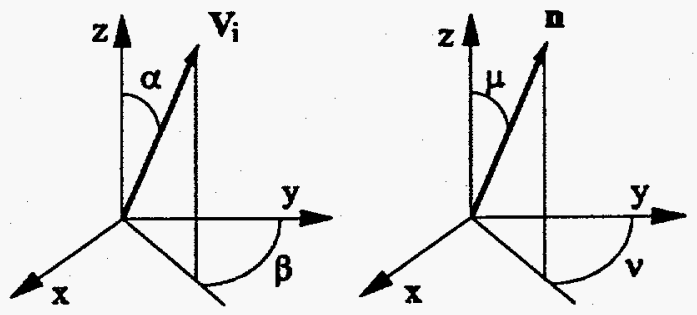

Fig. 2 Vectors $\mathbf{V}_{\mathrm{i}}$ and $\mathbf{n}$ in the coordinates 


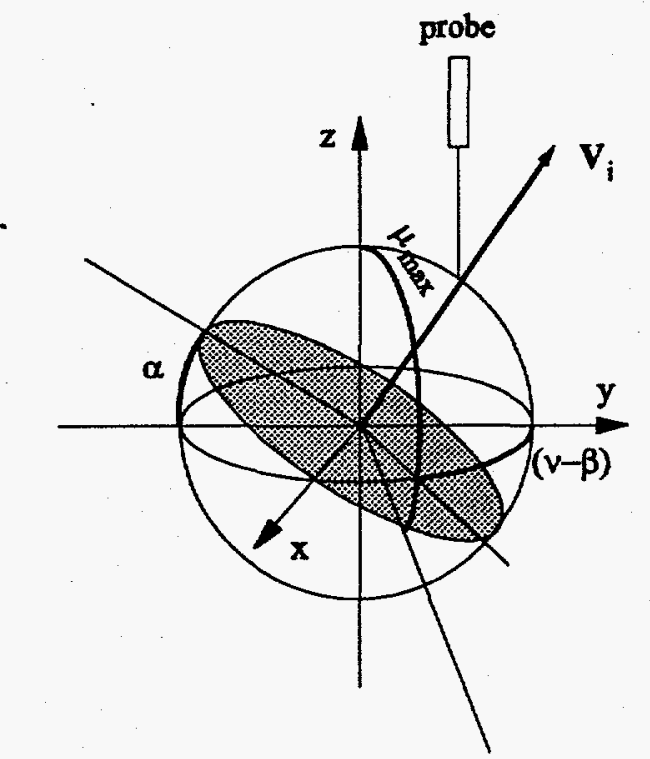

Fig. 3 Illustration of probe-bubble interaction and the definitions of angles 


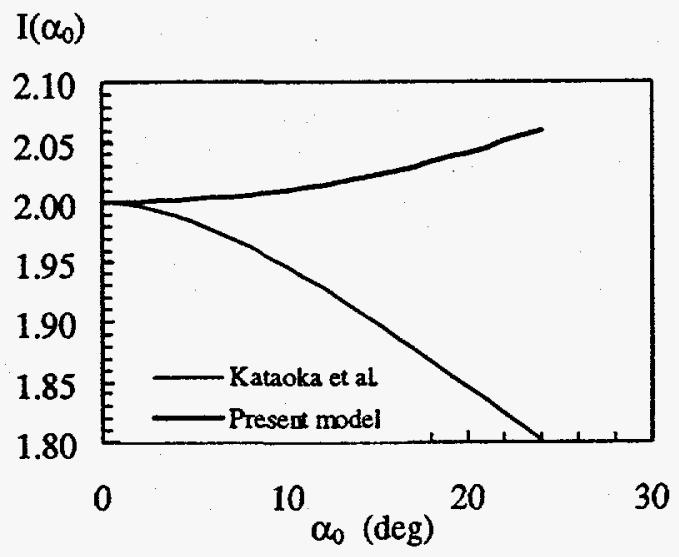

Fig. 4 Numerical results of Kataoka et al. and present analysis 


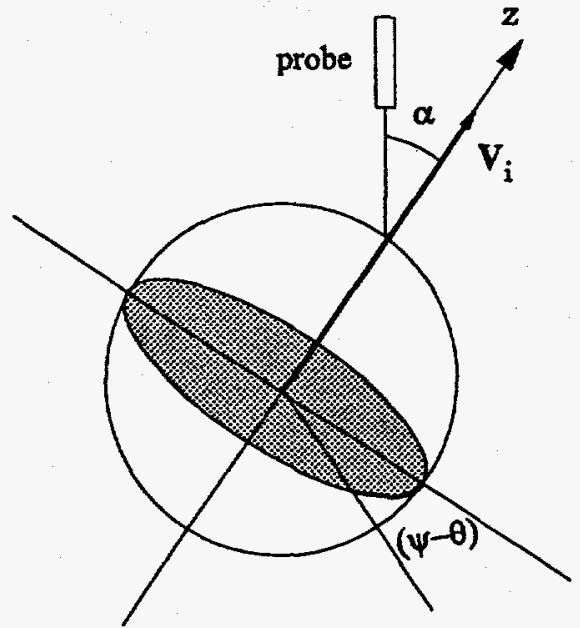

Fig. 5 Orientation of the new coordinate system 


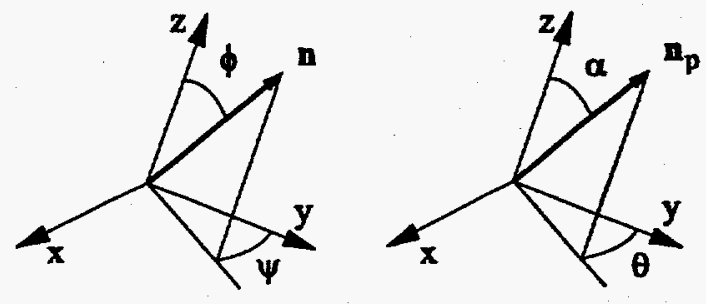

Fig. 6 Vector $\mathbf{n}$ and $\mathbf{n}_{\mathrm{p}}$ in the new coordinates 


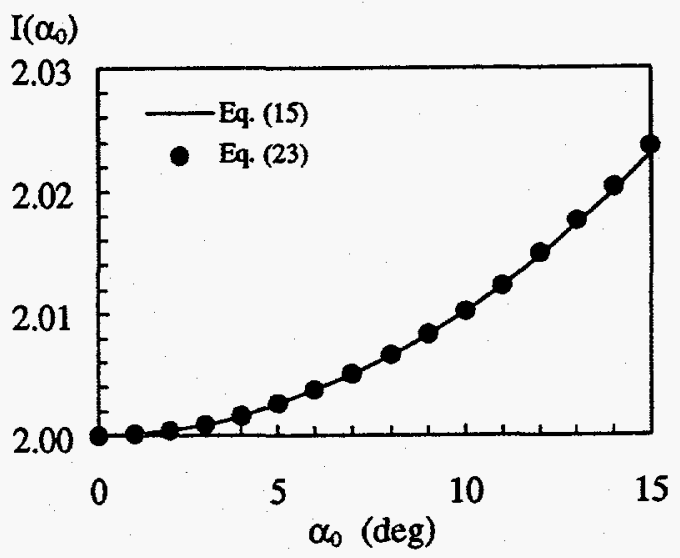

Fig. 7 Comparison of the integrations from the two suggested methods 